AUTOR: Óscar Rueda y Ma José Pizarro.

UNIVERSIDAD: ETSA de Madrid, Escuela de Arquitectura de la UEM.

BREVE BIOgRAFÍA: María José Pizarro y Óscar Rueda son arquitectos, doctores y Profesores Asociados de Proyectos Arquitectónicos en las Escuelas de Arquitectura de la ETSAM y la UEM. La actividad principal que desarrollan son los concursos de arquitectura, trabajando en paralelo en labores docentes, de investigación y publicaciones.

TÍTULO: Bekleidung: Gottfried Semper y la técnica textil como origen de la envolvente en la arquitectura.

TITLE: Bekleidung: Gottfried Semper and the textile technique as the origin of the spatial enclosure in architecture.

RESUMEN: A mediados del siglo XIX, Semper dejó escrito El principio de la vestimenta en la arquitectura - Das Prinzip der Bekleidung in der Baukunst-, enmarcado dentro su obra cumbre, El estilo. Con este texto argumentaba que la construcción se inició a partir de las técnicas textiles y, por tanto, que las envolventes en la arquitectura y sus patrones decorativos procedían de esta técnica y así se habían perpetuado a lo largo de la historia. Las repercusiones de esta teoría serían extraordinarias pues de esta manera Semper entregaba el protagonismo en la creación arquitectónica a la envolvente espacial, rompiendo con una larga tradición que otorgaba el protagonismo a la estructura perpetuada en los órdenes clásicos. Abrió de esta manera la puerta a nuevas formas de entender la envolvente en la arquitectura, que asumía desde ese momento autonomía y capacidad para transmitir códigos en el entorno de igual forma que los trajes y los vestidos lo permiten entre las personas, así como para ganar ligereza e independencia del soporte permitiendo incorporar nuevas tecnologías y materiales más acordes con los nuevos tiempos que se avecinaban. Con esta teoría Gottfried Semper sentaba las bases de una nueva arquitectura que se estaba gestando y eclosionaría en los albores del siglo XX.

ABSTRACT: In the middle of the Igth century, Semper wrote The Principle of Dressing in the Art of Building -Das Prinzip der Bekleidung in der Baukunst-, framed inside his greatest work, The style. With this text, he established that the beginning of construction came from the textile techniques and, therefore, that the spatial enclosures in architecture and his decorative patterns came from this technique and it had been perpetuated along the history. The implications of this theory would be extraordinary because Semper handed the protagonism to the spatial enclosure, breaking with a long tradition that gave prominence to the structure perpetuated in the classical orders. He opened the door to new ways of understanding architecture envelope, that assumed since then autonomy and the ability to transmit codes in the environment in the same way that dressings and suits permitting it between the people. As well as to gain lightness and independence from bearing and so allowing to incorporate new technologies and materials more consistent with the changing times. Gottfried Semper laid the foundation for a new architecture that was being developed and would be born in the early twentieth century.

PALABRAS CLAVE: Semper, Bekleidung, Envolvente, Textil, Vestimenta.

KEYWORDS: Semper, Bekleidung, Spatial enclosure, Textile, Dressing.

CONTACTO: oscar.rueda@uem.es 


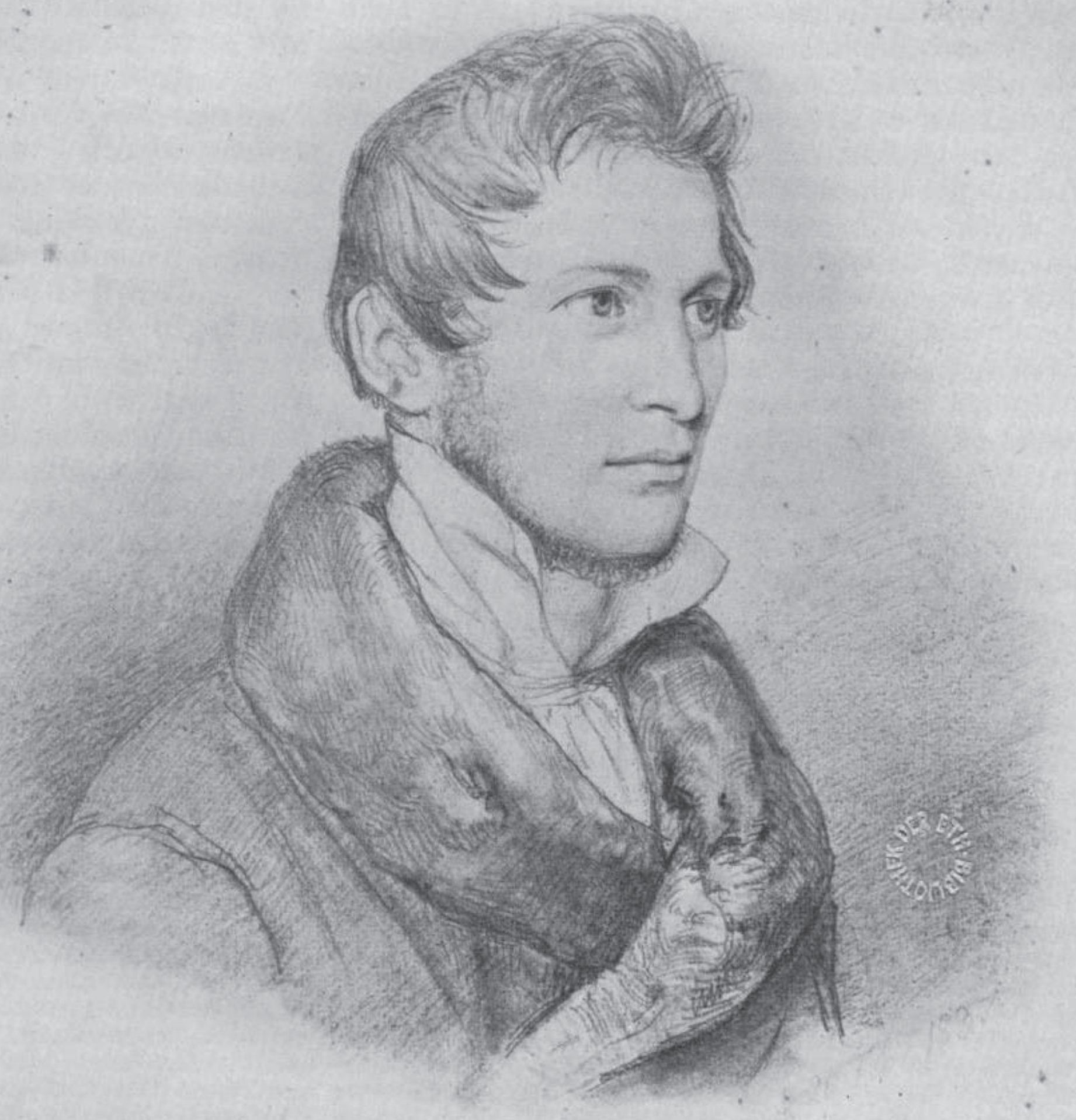




\section{BEKLEIDUNG: GOTTFRIED SEMPER Y LA TÉCNICA TEXTIL COMO ORIGEN DE LA ENVOLVENTE EN LA ARQUITECTURA}

Óscar Rueda y Ma José Pizarro

\begin{abstract}
"El arte de vestir la desnudez del cuerpo es, probablemente, una invención más temprana que el uso de cubiertas para delimitar el espacio. Hay tribus que, aún estando en un estado de barbarie muy primitiva y aunque no estén familiarizadas con el uso de la ropa, usan pieles e incluso poseen una industria más o menos desarrollada de hilado, trenzado y tejido para equipary defender sus campamentos... lo cierto es que el inicio de la construcción coincide con el inicio de los textiles."
\end{abstract}

Gottfried Semper (I803-I879) fue un arquitecto de gran reputación en el siglo XIX. Proyectó obras singulares tanto para la corte sajona de Dresde, donde entre I835 y I849 levantó su famoso Hoftheater, como para la corte de los Habsburgo en Viena, donde entre I869 y I879 dejó iniciados los Museos de Historia del Zwinger Imperial que completó su socio austríaco Karl von Hasenauer. En su época, era considerado como el arquitecto alemán más importante de la segunda mitad del siglo XIX, continuador del legado de Schinkel.

Pero sin duda lo que resultaría más trascendente en su trayectoria sería la elaboración de una singular teoría, muy bien argumentada y ampliamente documentada, en la que establecía que el inicio de la construcción procedía de las técnicas textiles y que, por tanto, los cerramientos en la arquitectura y sus patrones decorativos procedían de esta técnica y así se habían perpetuado a lo largo de la historia. A esta teoría la denominó El Principio de la Vestimenta en la Artquitectura -Das Prinzip der Bekleidung in der Baukunst-, y sentó los cimientos de la arquitectura moderna que se estaba gestando y eclosionaría en los albores del siglo XX.

La intuición de Semper al entregar el protagonismo arquitectónico a la Vestimenta -Bekleidung- en la arquitectura rompía con una larga tradición arquitectónica y cuestionaba los ideales del clasicismo vitruviano que había condicionado toda la producción arquitectónica hasta ese momento, académicamente instaurada y perpetuada en los órdenes clásicos. A la clásica tríada vitruviana utilitas, firmitas y venustas, con el protagonismo asumido por una estructura canonizada en los órdenes clásicos, Semper antepone una envolvente ligera, delimitadora del espacio, que asume el papel principal en la definición arquitectónica donde la estructura está subordinada y adquiere un papel secundario como mero soporte.

La herramienta principal que utilizará Semper en su teoría será la analogía textil para indagar en la relación entre vestimenta y envolvente arquitectónica. Y esta feliz analogía abrirá la puerta a nuevas formas de entender la envolvente en la arquitectura, que asumía desde ese momento autonomía y capacidad para transmitir códigos en el entorno de igual forma que los trajes y los vestidos lo permitían entre las personas. Así como para ganar ligereza e independencia del soporte permitiendo incorporar nuevas tecnologías y materiales más acordes con los nuevos tiempos que se avecinaban. Será en la Viena finisecular donde se destilen estas teorías y donde se pongan a disposición de la arquitectura moderna a través de Otto Wagner y Adolf Loos fundamentalmente, no sólo desde su propia práctica arquitectónica, sino también desde sus textos teóricos.
I.

SEMPER, Gottfried, Der Stil in den technischen und tektonischen Künsten, oder Praktische Äesthetik: Ein Handbuch für Techniker, Künstler, und Kunstfreunde. 2 vols.

(Frankfurt : Verlage für Kunst und Wissenschaft, I860-63), P. 247. Edición en inglés a cargo de Harry F. Mallgrave, Michael Robinson, Style in the Technical and Tectonic Arts; or, Practical Aesthetics. (Los Angeles, CA: Getty Research Institute, 2004). Con esta cita comienza Semper su famoso capítulo sobre El Principio de la Vestimenta en la Artquitectura -Das Prinzip der bekleidung in der Baukunst-. Traducción libre de los autores de este texto. 
2.

SEMPER, Gottfried, Vorläufige Bemerkungen über bemalte Architectur und Plastik bei den Alten. (Altona: Johann Friedich Hammerich Verlage, I834). Edición en inglés a cargo de Harry F. Mallgrave, Wolfgang Hermann Preliminary Remarks on Polychrome Architecture and sculpture in antiquity, en el libro The four elements of architecture and other writings, (Cambridge: Cambridge University Press, I989), P. 45-73.

3. SEMPER, Gottfried, Die vier Elemente der Baukunst (Braunschweig: Friedrich Vieweg und sohn, I85I). Edición en inglés a cargo de Harry F. Mallgrave, Wolfgang Hermann Preliminary Remarks on Polychrome Architecture and sculpture in antiquity, en el libro The four elements of architecture and other writings, (Cambridge: Cambridge University Press, I989), p. 75-I29.

4.

SEMPER, Gottfried, Ibid. The four elements... p. IO2.

5 .

LAYARD, A. H. Nineveh and its Remains. (London: John Murray, I849). Emille Botta y August H. Layard creen en un principio, ambos, ser los descubridores de la mítica Nínive. Sin embargo, el primero descubre Khorsabad y el segundo Nimrud.
Pero volvamos a lo que aquí nos interesa: la gestación de este principio teórico del origen textil de la arquitectura. Semper tuvo una formación arquitectónica clásica. Tras un breve paso por la Universidad de Gotinga, decide trasladarse a París donde estudia en la École des Beaux-Arts. Pronto emprende su viaje formativo a Oriente donde permanecerá hasta finales de I833. A su regreso vistará Berlín, donde se entrevista con Schinkel. Esto será crucial en su formación, pues a partir de ese momento se ve proyectado al centro de la mayor polémica que ocupaba la escena arquitectónica europea: la policromía en la arquitectura. Schinkel, ferviente neogriego, queda muy gratamente sorprendido por este joven aventurero que le enseña un manuscrito y unas láminas donde defiende que los templos griegos estaban policromados. Esto le anima a la publicación de su primer libro en I834, Notas preliminares sobre la policromía en la Arquitectura y escultura de la Antigüedad ${ }^{2}$, que le granjeará un notable prestigio y le abrirá las puertas de la Universidad de Dresde.

Pero no será hasta I85I cuando Semper destile su corpusteórico en una catarsis personal. Exiliado en Londres, arruinado y separado de su familia como consecuencia de su participación en la revolución liberal de I849 en la corte de Dresde, con su brillante carrera arquitectónica y docente arrojada por la borda, decide poner en limpio sus intuiciones teóricas en un breve manifiesto: su revelador texto Los cuatro elementos de la arquitectura ${ }^{3}$.

La primera mitad del libro de Los cuatro elementos es una prolongación de su ensayo anterior de Notas preliminares..., aportando nuevos datos para reforzar su teoría y rebatiendo punto por punto las tesis del influyente profesor de la Academia de Arte de Berlín Franz Theodor Kugler, que irónicamente le denominaba The red Semper y le tachaba de extremista, no solo en su posicionamiento político sino también teórico en su defensa de la policromía.

Sobre esa base elaborará su novedosa teoría y, en un giro espectacular, en la segunda parte del libro enunciará su tesis sobre el origen de la arquitectura. En un análisis "...antropológico de las condiciones primitivas." 4 , analiza los primeros asentamientos humanos llegando a una conclusión emparentada con los textos de Vitruvio: la primera huella de los asentamientos humanos fueron los restos del fuego en torno al que se reunían los hombres primitivos, el hogar. Para Semper, este era el elemento primigenio en torno al cual se fueron levantando los demás para protegerlo de la intemperie: la plataforma, el techo y la envolvente exterior. Y a cada uno de estos cuatro elementos se le asoció, desde tiempos remotos, una técnica y un material: cerámica para el hogar, cantería para la plataforma, carpintería para la cubierta y textiles para las envolventes. Pero para Semper, sin duda, el elemento más importante era la envolvente de origen textil pues contenía la esencia de la arquitectura: delimitar y, por tanto, crear el espacio.

Sin embargo, paradójicamente, la confirmación de su teoría la encontraría en descubrimientos arqueológicos posteriores. Camino del exilio, Semper pasa en París unos meses decisivos. En el verano de I849 gracias a Charles Blanc, director del Louvre y buen amigo suyo, se le abren las puertas de los depósitos del museo. Allí tuvo la oportunidad de examinar por vez primera los colosales paneles de alabastro procedentes de los yacimientos de mítica ciudad de Nínive, descubiertos en I842 por Emille Botta ${ }^{5}$. Eran los primeros vestigios encontrados de una civilización, la Asiria, de la que sólo se tenía noticia por relatos ancestrales como las sagradas escrituras. 

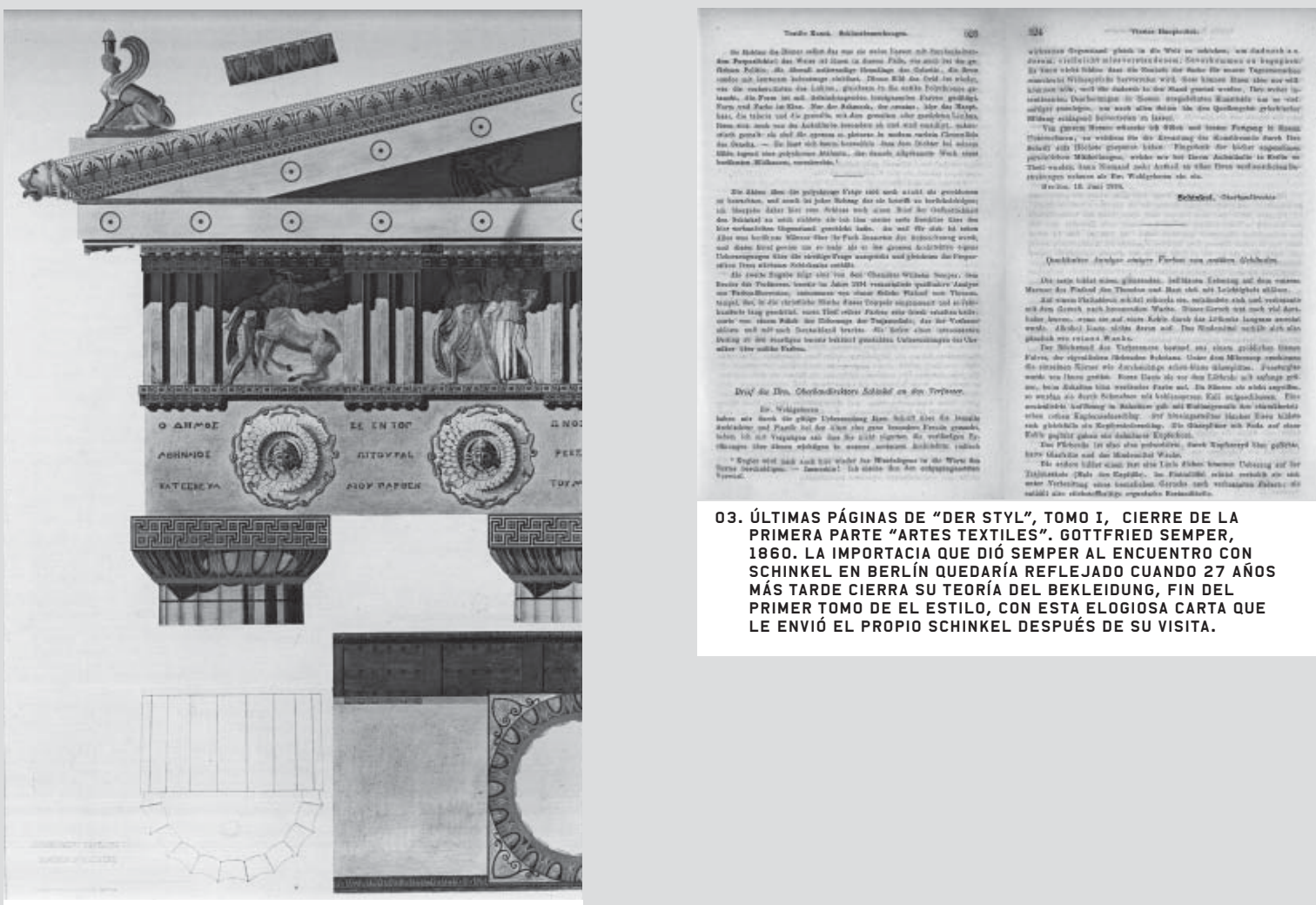

03. ÚLTIMAS PÁGINAS DE "DER STYL", TOMO I, CIERRE DE LA PRIMERA PARTE "ARTES TEXTILES". GOTTFRIED SEMPER, 1860. LA IMPORTACIA QUE DIÓ SEMPER AL ENCUENTRO CON SCHINKEL EN BERLÍN QUEDARÍA REFLEJADO CUANDO 27 AÑOS MÁS TARDE CIERRA SU TEORIA DEL BEKLEIDUNG, FIN DEL PRIMER TOMO DE EL ESTILO, CON ESTA ELOGIOSA CARTA QUE LE ENVIÓ EL PROPIO SCHINKEL DESPUÉS DE SU VISITA.

02. RECONSTRUCCIÓN DE LA POLICROMÍA DEL

ENTABLAMENTO DEL PARTENÓN.

ENERO DE 1832. GTA 215-1-3.

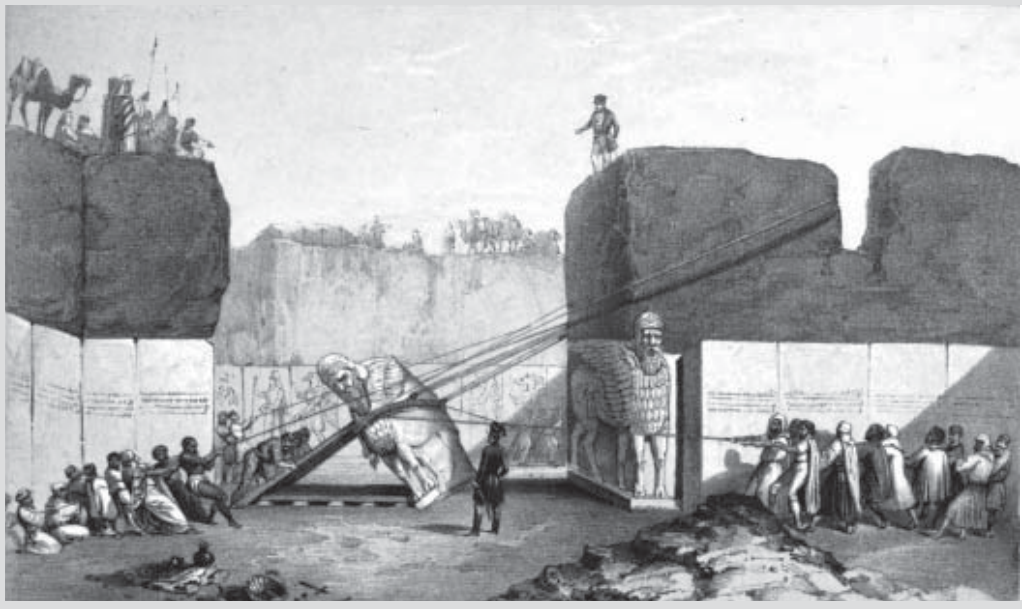

04. DESMONTAJE DIRIGIDO POR LAYARD EN 1847 DE LOS BAJORELIEVES DE ALABASTRO DE NINIVE EXPUESTOS EN EL PALACIO DE CRISTAL EN LA
EXPOSICIÓN UNIVERSAL DE 1851.

LÁMINA PROCEDENTE DE LA PUBLICACION: A POPULAR ACCOUNT OF DISCOVERIES AT NINEVEH. LAYARD, A. H. 1851, LONDON: JOHN MURRAY. EN ESTE LIBRO DESCRIBE CON TODO DETALLE LA PERIPECIA DEL DESMONTAJEY PITER R TRANSPORTE DE ESTAS EMILS QUETA EXAMINADAS POR SEMPER EN EL LOUVRE, FORMARÁN "THE NINEVAH COURT" IN THE CRYSTAL PALACE. EL COLOSAL BAJO RELIEVE DEL TORO ALADO EXPUESTO EN LONDRES QUE MEDIA 47 METROS DE ANCHURA POR 10 METROS DE LONGITUD APROXIMADAMENTE.

\section{Bon} (6) 可

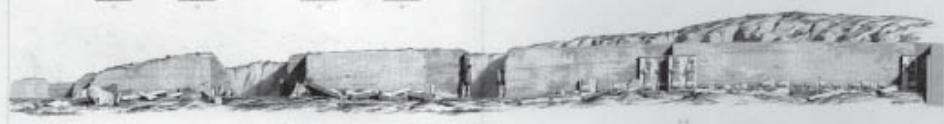

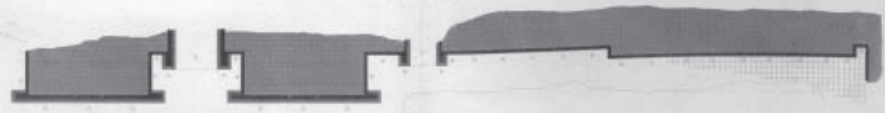

05. DESMONTAJE DIRIGIDO POR LAYARD EN 1847 DE LOS BAJORELIEVES DE ALABASTRO DE NINIVE EXPUESTOS EN EL PALACIO DE CRISTAL EN LA EXPOSICIÓN UNIVERSAL DE 1851.

DETALLE DE LOS MISMOS Y PLANO DE LA EXCAVACIÓN DE NINIVE, CON ALABASTRO, DIBUJADO POR P. E. BOTTA EN MONUMENT DE NINIVE, TOMO I, LAM. 30. 
6.

SEMPER, Gottfried, Ibid. Der Stil...

7.

SEMPER, Gottfried, Ibid. Der Stil... p. 217 edición alemana, p. 242 edición inglesa.

8.

SEMPER, Gottfried, Ibid. Der Stil... p. 233 edición alemana, p. 250 edición inglesa. Semper usa este término por primera vez al inicio del capítulo dedicado a Das prinzip der Bekleidung, donde lo define.
La materialidad de los paneles asirios y los motivos ornamentales de los mismos, que Semper identifica con la técnica de los tapices, le lleva a utilizar por primera vez el término Bekleidung -Vestimenta- en sus escritos. Pues bien, ante sus ojos estaba la confirmación palpable de sus teorías: la transmutación de paredes textiles en panelados pétreos, de los tapices orientales en láminas de alabastro tallado que vestían la arquitectura asiria sobre un soporte o subestructura que los sustentaba que carecía de relevancia.

Semper abandona París a finales de 1850 rumbo a Londres. Allí pasará cinco años en el exilio parapetado en sus teorías, trasladando su escritorio a la biblioteca del British Museum. Y dejará escrito su monumental tratado El Estilo, ${ }^{6}$ publicado en I860, donde desarrollará de manera exhaustiva las intuiciones de su manifiesto Los cuatro elementos. Decidió dividir el tratado en dos volúmenes: el primero dedicado al estudio histórico-artístico y técnico de cada uno de los cuatro elementos, la parte intrínseca de cada uno de ellos; el segundo a los condicionantes externos, sociales, ambientales, culturales, la parte extrínseca. El primer volumen debía iniciarse con la cerámica, puesto que el hogar era el motivo fundacional de la arquitectura. Pero los problemas con su editor E. Vieweg originan la pérdida del manuscrito con el capítulo de la cerámica ya terminado. Al cambiar de editor, Semper se centra en el capítulo del Arte Textil, cuyo borrador tenía más avanzado y mantenía en su poder. Y aquí se produce el cambio más relevante para el tema que estamos tratando: en la segunda parte del capítulo del Arte Textil, dedicado a la técnica, incluye un añadido de 300 páginas que denomina Das Prinzip der Bekleidung in der Baukunst -El principio de la vestimenta en el arte de la construción- ${ }^{7}$ pasando de 220 a 520 páginas. Esto origina que en I860 divida el primer tomo, Teoría Artísticay Técnica, en dos volúmenes y sólo aparezca el Arte Textil en el primero de ellos, publicando el segundo volumen en I863 dedicado a la Cerámica, la Carpintería (Tectónica) y la Mampostería (Estereotomía). Añade un apartado dedicado a la Metalurgia, que considera un arte híbrido pero relevante. El enfoque del texto tendrá un marcado carácter antropológico, como ya comentamos, pero apoyado en dos herramientas muy potentes incorporadas por Semper: el análisis científico y etimológico.

Para el análisis científico Semper se inspiró en la evolución de las especies, en una analogía biológica que partía de dos conceptos fundamentales: primero los motivos primordiales -Urmotiven-fijados por Semper en dos arquetipos, el hogary la tela -Urherdy Urtuch-, las dos primeras huellas de los asentamientos humanos; y en segundo lugar, la Stoffwechsel -Metamorfosis o Transfiguración de la materia- ${ }^{8}$ en las artes aplicadas, que es el mecanismo por el cual un Arquetipo cambia de materialidad debido a un tratamiento técnico para adaptarse a unos nuevos condicionantes culturales pero manteniendo sus propiedades artísticas y simbólicas originales.

De esta manera, para justificar el origen antropológico del arte textil, escogerá relatos etnográficos que describen el trenzado o tejido de esteras fabricadas con el fin de colgarlas verticalmente y definir un recinto. Y continuará hasta la etapa clave en la que consideró se había alcanzado la perfección evolutiva de la técnica textil: la antigua Asiria, famosa por sus coloridas tiendas de campaña y sus tapices. La necesidad, origen verdadero del arte para Semper, llevó a los asirios a construir paredes más sólidas, que vistieron con ricos tapices y que se transfiguraron más tarde en vestidos alternativos de estuco, empanelados de madera, placas de metal, revestimientos de terracota o paneles de alabastro. Pero siempre manteniendo la esencia del motivo original textil: la vestimenta-Bekleidung-seguía siendo el elemento definidor del espacio, frente al soporte que permanecía oculto y tenía un carácter secundario. 

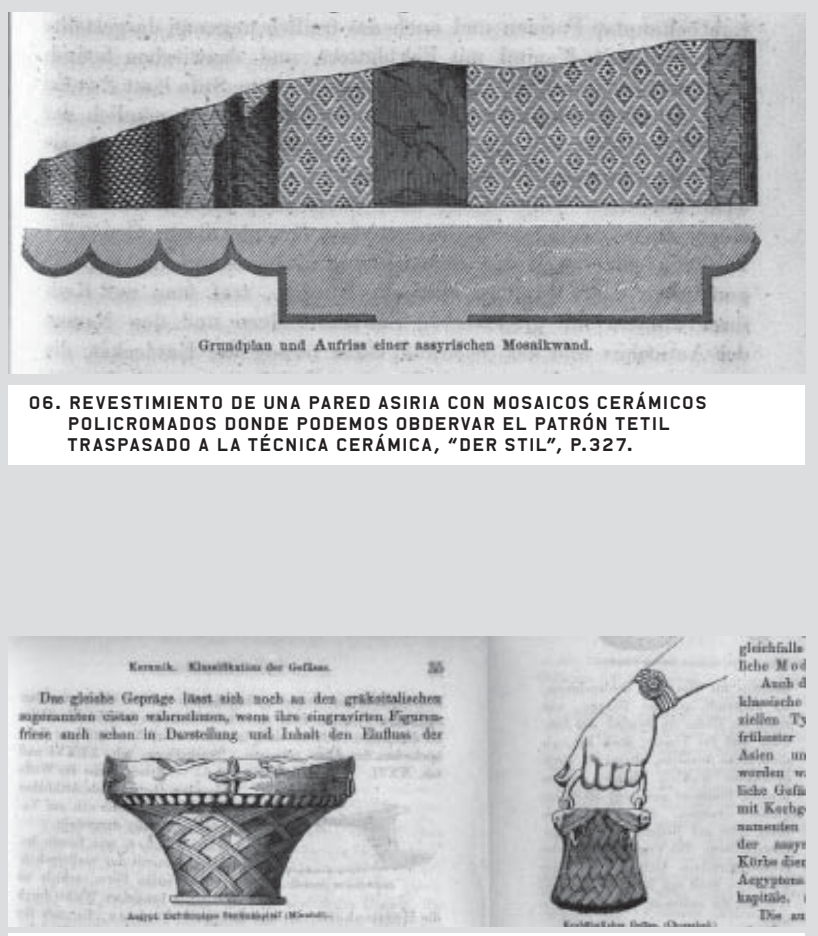

08. CESTOS USADOS COMO MOTIVOS EN CAPITELES EgIPCIOS. "DER STYL", P. 35 II VOL.

1 Dieser-Realismus tritt $\mathrm{z}$. der Säulenschäfte. Die Zahl de

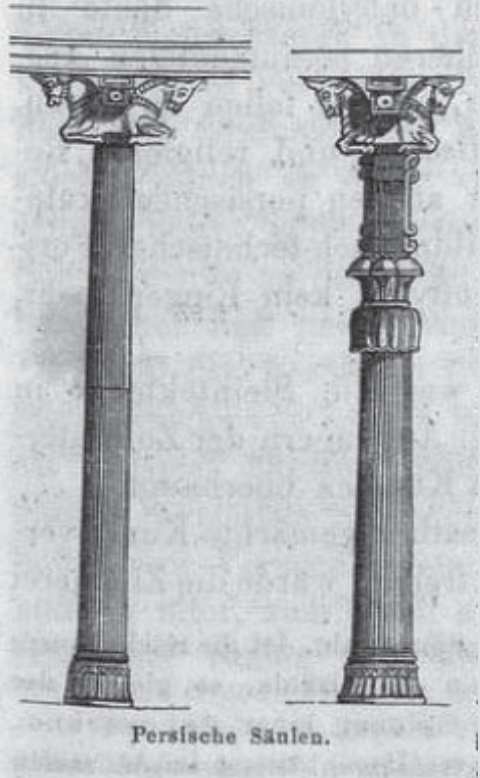

(Bd.I. \$. 68 bes. S. 384 u. 84 n., ff.). Steintektonik, als zu möbelhaft, $g$ behält, nämlich den Vasenunters:

10. COLUMNAS ASIRIAS DE "CUERPO HUECO" (VESTIDO DE BRONCE SOBRE NÚCLEO PORTANTE DE MADERA) Y ESQUEMA DE ORIGEN DESCONOCIDO UTILIZADO POR CONSTRUCCIÓN ASIRIO, "DER STYL", P. 399 VOL II Y P. 412 VOL. I.

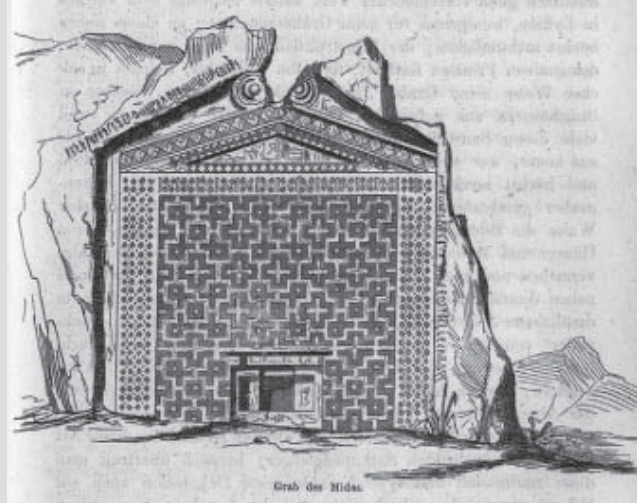

07. TUMBA DE MIDAS, ILUSTRANDO PATRONES TEXTILES PETRIFICADOS, "DER STYL", P. 429.

Ein direkter und materieller Zusammenhang zwischen dem Kostümwesen und der Plustik tritt ż. B. in dér Thatsache zur Evidenz, dass die uralte Sitte des Ankleidens der hölzernen Kult-
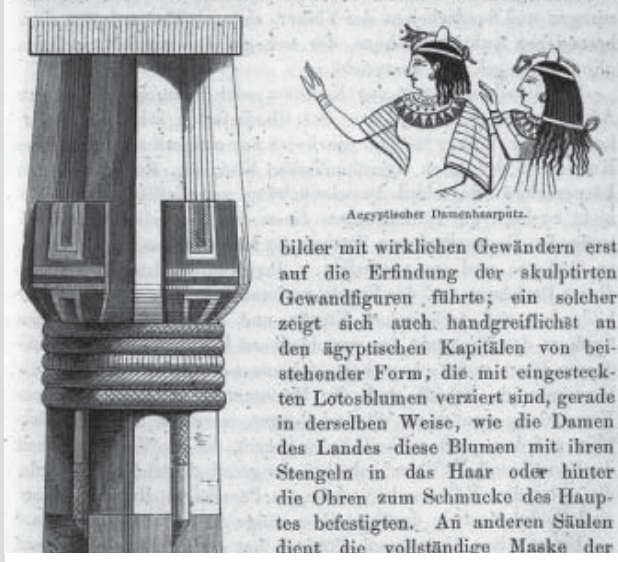

bilder mit wirklichen Gewändern erst auf die Erfindung der -akulptirten Gewandfiguren fuihrte; ein solcher zeigt sich such. handgreiflichst an den agyptisehen Kapitälen von beltehender Form die ant - eingesteck-

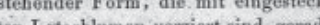
ten Lotosblan Werace

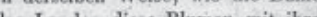
des Landes diese Blamen mit ihre Stengeln in das Haar oder hinte die Ohren zum Schmueke des Hauptes befestigten. Añ anderen Situlen

09. ORNAMENTOS TEXTILES EGIPCIOS PARA EL PELO, TRASLADADOS A LOS CAPITELES DE LAS COLUMNAS, "DER STYL", P. 211

Textile Kunst. Assyrien.

in das innerste Gesetz der Natur geblickt organischen Gebilde nach dem Röhrensyste

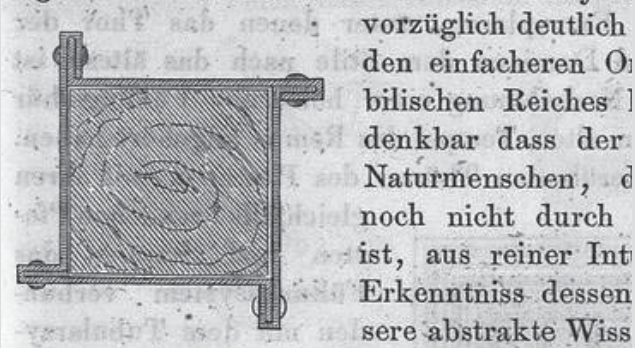

11. COLUMNAS ASIRIAS DE "CUERPO HUECo" (VESTIDO DE BRONCE SOBRE NÚCLEO PORTANTE DE MADERA] Y ESQUEMA DE ORIGEN DESCONOCIDO UTILIZADO POR ASME "DAR STYL", P. 
9.

HUMBOLDT, Wilhelm von. Über die Verschiedenheit des menschlichen Sprachbaus und seinen Einfluss auf die geistige Entwicklung des Menschengeschlechts-Sobre las diferencias de la estructura del lenguaje humano y su influencia sobre el desarrollo intelectual de la humanida- (Berlin: F. Dümmler Verlage, I836), p. 4I. La cita dice así: "Sie selbst ist Kein Werk (Ergon), Sondern eine Thätigkeit (Energeia)"

IO.

SEMPER, Gottfried, Ibid. Der Stil... P. 77 edición alemana, P. I53 de la edición Inglesa.

II.

SEMPER, Gottfried, Ibid. Der Stil... P. 79 edición alemana, P. I64 de la edición Inglesa. Nota I3 extensa a pie de página.

I2.

SEMPER, Gottfried, Ibid. Der Stil... P. 229 edición alemana, P. 248 de la edición Inglesa.
Pero quedaba la hipótesis más arriesgada: concluía que la policromía en el arte oriental había surgido de los antiguos telares de los asirios. Y se había trasladado desde allí a la arquitectura de la antigua Grecia, donde se encontraba el ideal de la belleza y la perfección evolutiva de este motivo, pues el sistema político griego había roto las cadenas de la teocracia y su arte era libre. Planteaba así la tesis de que la policromía griega, que era la que de verdad le interesaba en su línea de razonamiento evolutivo, tenía su génesis histórica en el acto primordial de hacer tapices y levantarlos después para construir paredes con ellos.

Con la siguiente herramienta, la etimología, Semper hizo verdaderos malabarismos inspirado por las tesis de Wilhelm von Humboldt y su concepción antropológica del Lenguaje. En su famoso libro Sobre las diferencias de la estructura del lenguaje humano... parafraseando la ética aristotélica escribía:

"... el lenguaje no es una obra -Ergon-, sino una actividad -Energeia-" 9

Es decir, el lenguaje no solo describe sino que vocaliza la acción, interpreta una voluntad. Para Humboldt, el lenguaje crea la estructura del pensamiento y cuando nombra algo deja una huella que contiene la esencia de las cosas. Esta concepción antropológica, que cede el protagonismo al lenguaje, será central en las argumentaciones de Semper que usará la etimología para justificar las raices históricas de las técnicas que analiza. Para cerrar la primera parte del arte textil, Semper escribe: ...la costura-Die Naht-, es la técnica primitiva que permite la unión de superficies distintas en un conjunto homogéneo. ${ }^{\text {Io }} \mathrm{Y}$ a continuación realiza una curiosa asociación de ideas:

"La costura expresa el principio de hacer de la necesidad una virtud... Naht -costura, unión-y Noth - necesidad- están relacionadas etimológica y conceptualmente. Una asociación de ideas similar existe entre Nahty Knoten (Unióny Nudo) [Latín: nodus (nudo), nexus (conexión)], y entre la ananke (necesidad, griego en original) y la necesidad (Noth), que no puede simplificarse, que puede interpretarse de distintas maneras, pero que no es fruto de la casualidad..."

Para a continuación citar a un discípulo de Humboldt, el Dr. Albert Höfer, que investigó la raíz indoeuropea noc: en latín nec-o, nexus, necessitatis, nectere y en griego neo, hilar. Es decir, para Semper Nudo y Costura (Knoten y Nath), comparten la misma raíz arcaica y a su vez procedían de Noht, Necesidad, el verdadero origen del Arte. Por tanto, la técnica primigenia era la costura generada a partir del nudo que era, por tanto, el elemento estructural básico, tectónico, del que deriva la técnica edificatoria a partir del arte textil.

Y esto enlazaba con otros términos ...prearquitectónicos, desde los que surgió la verdadera arquitectura. ${ }^{12}$ Esos términos eran Wand-pared-y Gewand -vestido- que comparten la misma raíz y el mismo significado en la antigüedad: envolvente espacial. Y otros como Decke -cubierta, también manto-, Bekleidung -revestimiento, también vestido-, Schranke -barrera, también ropero-, Zaun-cercado, similar a Saum que es dobladillo-, lo que justificaba el origen textil de estos términos técnicos de la construcción. En definitiva, habríamos llegado a lo que denominamos El origen textil de la arquitectura: ... el inicio de la construcción coincide con el inicio de los textiles. 


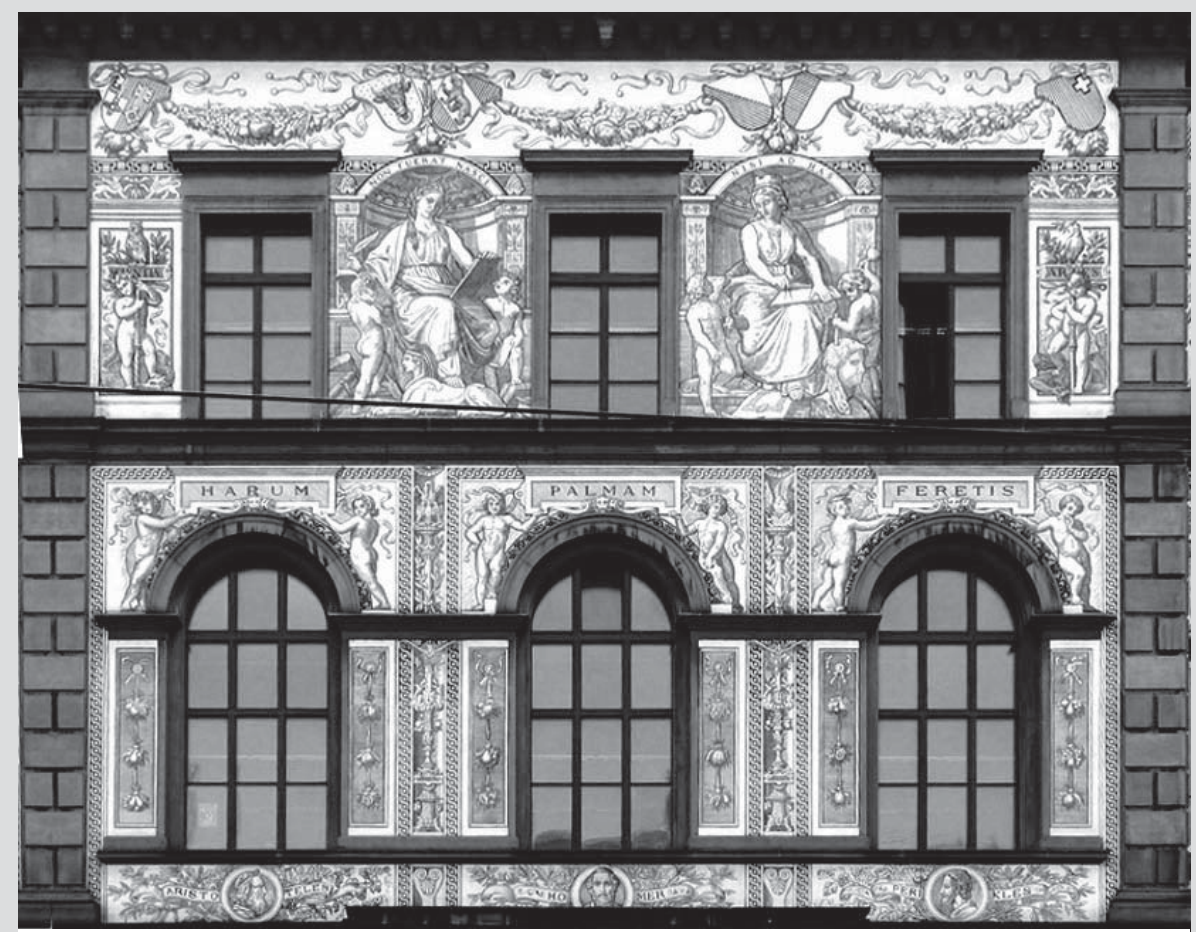

12. GOTTFRIED SEMPER, ETH ZURICH (1859-1863), DETALLES DE LOS DIBUJOS DE LA FACHADA NORTE. FOTOGRAFÍA DE LA FACHADA NORTE, ESTADO ACTUAL RESTAURADA. FOTO ACTUAL DEL AUTOR.

enden und sẹine Festigkeit begründet sich hauptsatchlich auf den Widerstand der Reibung. Das System, welches durch Seitendruck die Reibung am meisten befördert, wenn die beiden Fäden in entregengesetyten Richtungen den, ist das festeste. Andere Verbälnisse tretén ein, wenn auf die Fäden nicht in dem

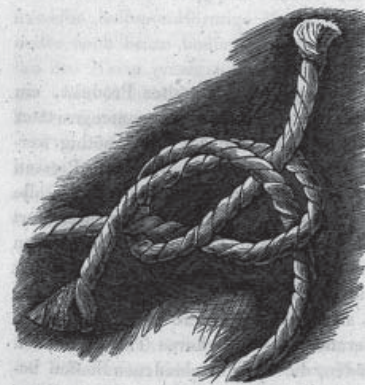
Sinne ihrer Lainge, sondern vertikal auf deren Ausdehnung eingewirkt wird, obgleich auch hier die nach der Lalngenrichtung der Fäden gehende Resultante der Spannum am meisten in Betrach Det Wetrach kommt. Der Weberknoen ist unter allen de festeste und nützlichste, vielleicht auch der alteste oder doch derjenige, der in den technischen Künsten am frihsten figurirte. Die Seiler und Schiffer kennen ich leider nur als. Laie sprechen könnte. Manches auch für unsere ich leider nur als. Laie sprechen könnte. Manches anch für unsero
' s. Wilkinson's oft eitirtes Werk über Aegypten. Vol. III. s. 144.

13. PÁGINA DE "DER STIL" QUE ILUSTRA LAS TÉCNICAS EL NUDO, SÍMBOLO TECTÓNICO PRIMORDIAL. P, 184 .

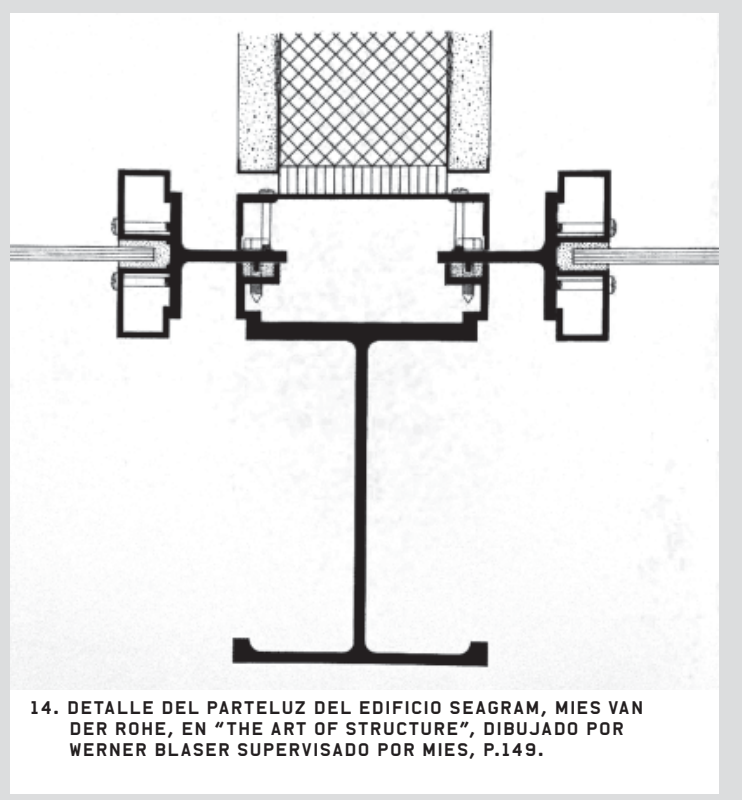


I3.

SEMPER, Gottfried, Ibid. Der Stil...p. 248 edición alemana, p. 760 de la edición inglesa.

I4.

SEMPER, Gottfried, Ibid. Der Stil... p. 444 edición alemana, p. 667 de la edición inglesa.
Anteriormente habíamos concluido que los Asirios desarrollaron este principio constructivo textil, que llegó primero a Egipto y más tarde a Grecia, pero que sufrió una gran metamorfosis con el Arte Helénico. Los asirios habían desarrollado un sistema a la vez constructivo y ornamental que desembocó en la diferenciación de un núcleo interior sustentante y un acabado exterior que contenía la esencia artística: muros cerámicos o de paneles de alabastro con un interior de material portante oculto, intrascendente; o los soportes verticales de los palacios ...columnas de madera vestidas de bronce, que son en sí mismas mástiles más o menos monumentales de una tienda de campaña. ${ }^{\text {I3 }}$

Sin embargo, los egipcios actuaron al revés que los asirios pues tallaron el vestido, que en realidad era la última capa de piedra sobre un núcleo sólido y masivo hecho del mismo material, sin un principio estructural: el vestido estaba unido al cuerpo interior, núcleo y superficie estaban trabados y tenían la misma materialidad. El arte helénico combinó ambas técnicas, empleando una mampostería regular tallada como la egipcia, de corte "isodómico", que ocultaba las juntas en el sentido simbólico y estructural asirio:

"...las formas del arte asirio basadas en necesidades mecánicas se transformaron en formas dinámicas, incluso orgánicas, una manera de dotarlas de alma ... Este nuevo estilo no necesitaba referencias al peso o a la inercia de la masa, se había emancipado del material. Era un ser vivo, y nunca preguntaríamos de que materiales está hecha una criatura y la calidad y cantidad de los mismos". ${ }^{14}$

Y este estilo arquitectónico "reivindicó el color como el vestido más sutil e incorpóreo". Parafraseando a Humboldt, la arquitectura pasaba de Ergon (obra o creación inerte) a Energeia (obra orgánica, dotada de alma). O usando la preciosa analogía empleada por Semper en su juventud en "Notas Preliminares...", percibimos el mamut como un ser vivo, con piel y músculos (el templo griego policromado) y no como un conjunto de huesos fosilizados (el templo griego como ruina romántica con el mármol blanco al desnudo).

Podríamos establecer, por tanto, una triple repercusión de la teoría del Bekleidung de Semper.

- La envolvente arquitectónica es la herramienta primordial para definir el espacio, desde tiempos inmemoriales, elevada a categoría artística por vez primera por los asirios. A partir de Semper, la escuela de pensamiento alemana empieza a centrarse en la noción de Espacio como esencia de la creación arquitectónica. Mientras Semper articula una teoría en la que la envolvente espacial de origen textil toma el protagonismo en la creación del espacio arquitectónico, no olvidemos que alguien tan avanzado en su pensamiento arquitectónico como Viollet-le-Duc encamina la escuela francesa hacia una línea estructuralista donde la noción de espacio no se menciona. A finales del siglo XIX eclosionará esta línea de pensamiento semperiana en la escuela alemana que desarrollará la noción de espacio moderno con pensadores como August Schmarsow, y su tratado sobre el espacio Esencia de la creación arquitectónica, o Theodor Lipps y su teoría de la Empatía estética (Einfühlung).

- El carácter sensitivo y significante de la envolvente: La percepción sensitiva de la envolvente, ligada a su materialidad, será central en la arquitectura germanica en el siglo XX, no sólo de Adolf Loos sino también en la de Mies van der Rohe. Ambos profundizarán en el placer sensitivo que produce la envolvente arquitectónica concebida como un traje, con un haz exterior significante y un envés interior táctil en contacto con el cuerpo. Plantearán un uso lúdico y cálido del espacio, en las antípodas del espacio frío y abstracto que se ha querido transmitir por la historiografía oficial. Una forma de habitar, de connotaciones nietzschianas, como 

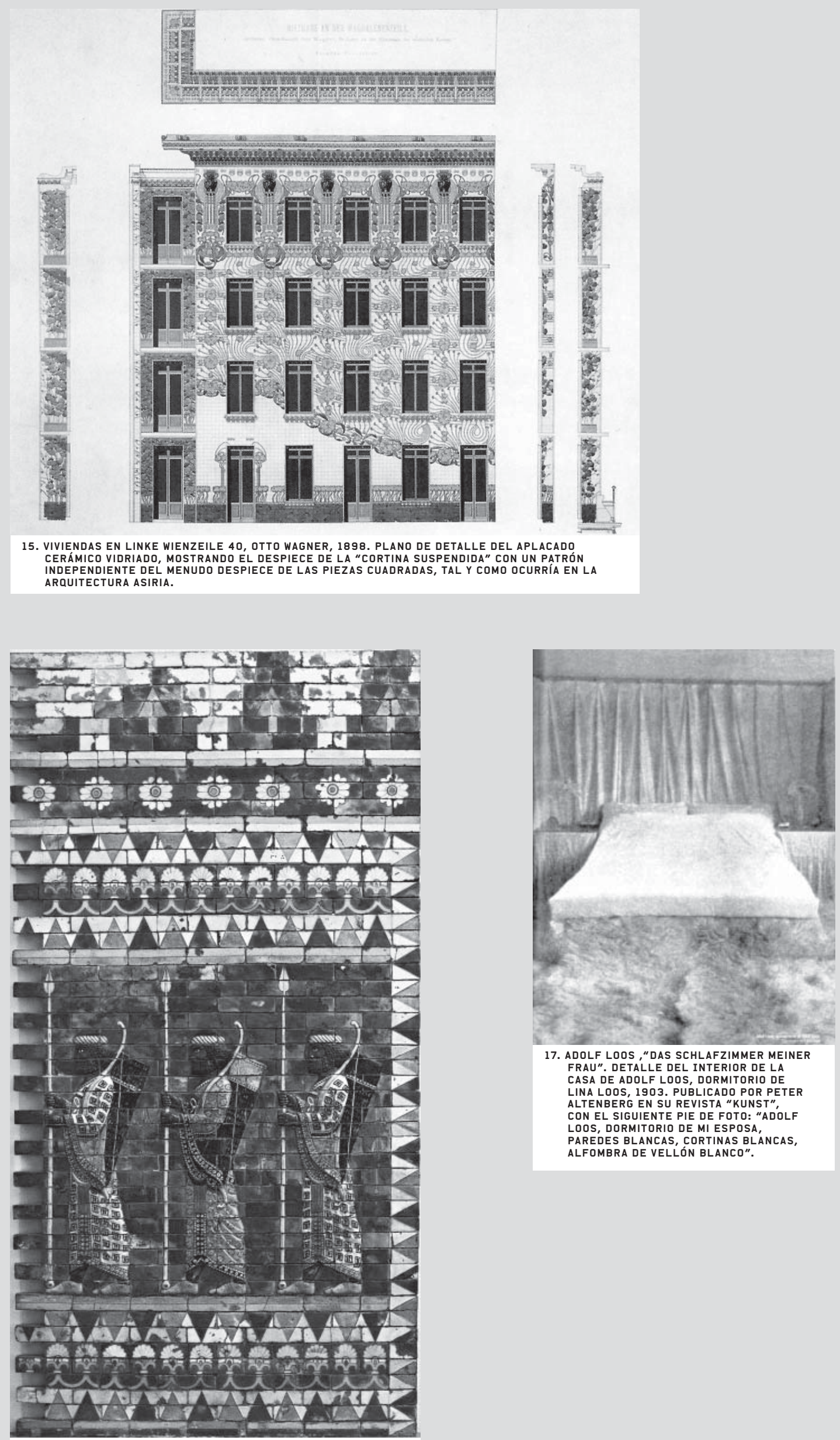
16. DETALLE DE LA CERÁMICA VIDRIADA QUE RECUBRía LA
PUERTA DE ISTHAR. FOTO ACTUAL DEL AUTOR. MUSEO DE
PÉRGAMO, BERLIIN. 
I5.

RIEGL, Alois. Die spätrömische Kunst-Industrie, (Viena: Österreich. Staatsdruckerei Verlage,I90I), p. 42. Arte industrial tardorromano, (Madrid, Ed. Visor, I992). expresión del gozo de la vida y afirmación del protagonismo del sujeto y, por tanto, de la empatía. Esta arquitectura llevará al límite las percepciones sensoriales tanto de materiales tradicionales como de nuevos materiales procedentes de la industria más avanzada tecnológicamente.

La noción de empatía incidirá extraordinariamente en la simbología de la envolvente, en la que tanto énfasis había puesto Semper. El carácter significante de la envolvente y los códigos que transmite al observador tomarán protagonismo en la obra de Otto Wagner y Adolf Loos simultáneamente, aunque con distinta formalización. Y para ello la noción de la autonomía de la envolvente frente a una estructura secundaria, velada, será fundamental.

- Por último, la insistencia en el uso del término Baukunst frente a Arkitectur, que como ya explicamos enfatiza la componente constructiva de la edificación donde la arquitectura, como una de las Bellas Artes, es solo una parte del conjunto. Este elogio de la técnica está muy vinculado a otro tema fundamental: el respeto por la tradición, por el conocimiento de los materiales que se han empleado a lo largo de toda la historia y las técnicas asociadas que se han utilizado. Tema que centrará la lucha intestina entre Loos y los inventos decorativos de sus coetáneos de la Secesión vienesa.

Pero quedaban algunos cabos sueltos en la teoría de Semper. Había una paradoja: al alcanzar su esplendor la técnica de la vestimenta en la arquitectura con los templos griegos policromados aún no se había desarrollado la noción de espacio, era un arte puramente visual donde la superficie exterior se concebía como un bajo relieve y no existía el espacio interior. Esta contradicción no estaba resuelta por Semper en su teoría al establecer que la envolvente define el espacio y, sin embargo, centrarse en tres civilizaciones como la asiria, la egipcia y la griega que no manejaban la noción de espacio. Será Riegl quien plantee esta dicotomía en su profundo y polémico análisis de la arquitectura tardorromana al sentenciar categóricamente que: el interior del Panteón es el primer espacio en la arquitectura, un espacio no puramente visual sino táctil. ${ }^{15}$

Y esta dicotomía semperiana entre interior y exterior, y sus contradicciones, quedarán resueltas con maestría definitivamente por Adolf Loos cuya arquitectura será concebida No como revestimiento sino como vestimenta-Bekleidung-, como una envolvente espacial con un haz y un envés, cada una con su significancia pero inseparables, sustentadas por un armazón oculto. Y en esto, Loos será radicalmente distinto de sus coetáneos de la secesión vienesa, centrados como Semper en la decoración de la superficie exterior tratada como un vestido. En conclusión, con el apoyo de sus textos críticos, Loos será quien complete y resuelva los temas pendientes de La teoría de la vestimenta -Bekleidung- de Semper y siente de esta manera las bases de la arquitectura moderna en el siglo XX:

“... pongamos que el arquitecto tuviera la misión de crear un espacio cálido y habitable. Las alfombras son cálidas y habitables. Este espacio se podría resolver poniendo una de ellas en el suelo y colgando cuatro tapices de modo que formaran cuatro paredes... Pero tanto la alfombra como el tapiz requieren un armazón constructivo... Concebir este armazón es la segunda misión del arquitecto. Este es el camino correcto, lógico y real que debe seguir el arte de la construcción (Baukunst). La humanidad aprendió a construir en este orden.

Lo primero fue la vestimenta (Bekleidung).” ${ }^{\prime 6}$ 